\title{
Balance hídrico de la cuenca Mbói Caé asociado a El Niño: oscilación del Sur mediante el sistema Hydro-BID
}

\author{
Verónica Berestovoy ${ }^{1}$
}

\begin{abstract}
Resumen
Introducción: La crisis del agua es un problema global y la misma no solo se centra en su disponibilidad como se cree habitualmente. La crisis de agua involucra tres factores generales: distribución, accesibilidad y calidad. La distribución porque existen regiones muy húmedas y muy secas, como ejemplo en Paraguay se tiene la región oeste occidental con un promedio de precipitación de $600 \mathrm{~mm}$ anuales y la región sureste oriental con más de 1800 $\mathrm{mm}$ anuales (DMH, 2015). En cuanto a la accesibilidad, se tiene poblaciones que no cuentan con recursos, infraestructura y monitoreo para acceder al agua potable. La calidad que se ve afectada por el escaso sistema de saneamiento y los diferentes tipos de contaminantes, todo esto, aunado a las consecuencias del cambio climático y al crecimiento demográfico de las poblaciones que repercuten negativamente al ambiente y a la sociedad; es por ello que la planificación de recursos hídricos constituye una de las principales herramientas para enfrentar esta problemática. El conocimiento de la estructura del balance hídrico de lagos, cuencas superficiales y cuencas subterráneas, es fundamental para conseguir un uso racional de los recursos de agua en el espacio y en el tiempo, así como para mejorar el control y redistribución de los mismos El área estudiada la conforman la cuenca del arroyo Mboi Caé del departamento de Itapúa, comprendiendo 4 distritos: Encarnación, Capitán Miranda, Cambyretá y Nueva Alborada. Para el desarrollo del trabajo se tuvieron en cuenta datos hidrometeorológicos de temperatura, precipitación y caudal y se simuló el comportamiento del balance hídrico en el periodo 2013 a 2016, ya que en estos años el ENOS se caracterizó como muy fuerte por su intensidad mediante el sistema Hydro-BID.
\end{abstract}

Objetivo: Evaluar la influencia del fenómeno climático El Niño-Oscilación del Sur (ENOS) en el balance hídrico de la cuenca del arroyo Mboi Caé y generar información útil para la Gestión Integrada de Recursos Hídricos.

Material y Método: Se utilizaron los programas: Excel, QGIS 2.10 Pisa como sistema de información geográfica e HydroBID como software para la modelación hidrológica. Los datos meteorológicos de precipitación y temperatura se obtuvieron de la Dirección de Meteorología e Hidrología (DMH)

1. Facultad de Ciencias y Tecnología. Universidad Nacional de Itapúa, Paraguay.

E-mail: vero.beres@gmail.com

DOI: 10.26885/rcei.foro.2017.12 
y de la Universidad del Este de Anglia (UEA Caudales. Los caudales (m3/s) fueron obtenidos de un proyecto llevado a cabo por la Comisión Mixta ParaguayoArgentina del río Paraná y del Observatorio del Agua. La AHD fue proveída por el Sistema HYDRO. La base de datos Sqlite fue proveída por el BID. El análisis de datos climáticos fue realizado mediante gráficos que permiten observar los diferentes valores que adquieren las variables para cada año desde 1970 hasta el 2016. Se utilizó el programa QGIS versión 2.10 Pisa para procesar la base de datos AHD, se identificó el área que ocupa la cuenca de estudio, las subcuencas y principalmente el COMID para poder utilizarlo posteriormente en HydroBID. Se instaló la herramienta AHD (AHD Tools) con el plugin AHDTools en QGis. Los datos de temperatura y precipitación fueron organizados en formato csv en los periodos 2013 a 2016 e tomando los valores de las estaciones de Encarnación y Capitán Miranda, para su interpolación en el sistema HydroBid que se realizó mediante la herramienta "Climate Data Interpolating Tool". El balance hídrico de la cuenca de los arroyo Mboi Caé fue obtenido mediante la simulación hidrológica en el sistema HydroBID

Resultados y Conclusiones: La influencia del fenómeno ha sido mayor en su fase inicial (año 2014). El Hydro-BID constituye una herramienta de modelación hidrológica útil para América Latina y El Caribe que permite simplificar el comportamiento del agua y clima. En cuanto a la temperatura los meses más cálidos son desde diciembre a febrero con una temperatura media entre 25 y $26{ }^{\circ} \mathrm{C}$, las máximas se dan mayoritariamente en diciembre alcanzando entre 40 y $42^{\circ} \mathrm{C}$. Los meses más fríos corresponden a junio y julio con una temperatura media de 15.5 y $16^{\circ} \mathrm{C}$.. En general, se tiene las últimas décadas como más cálidas respecto a las temperaturas máximas y mínimas alcanzadas. En cuanto a la precipitación el promedio es de $1750 \mathrm{~mm}$ anual y los valores máximos oscilan entre un 50 a $60 \%$ más de dicho promedio, mientras que los mínimos un 12 a 38\% menos. El año 2014 fue el año más notable en cuanto a cambios en el balance hídrico relacionado con el aumento en la disponibilidad de agua, siendo uno de los más lluviosos desde 1970. Se puede notar que se tiene un volumen importante de agua que fluye hacia las aguas subterráneas y superficiales por lo que resulta importante un control y monitoreo de la calidad de la misma. El sistema HydroBID, es una herramienta sumamente eficaz para obtener información útil para la planificación de los recursos hídricos. Es adaptable a toda la región y están disponibles mediante el BID. Los resultados obtenidos en Hydro-BID pueden ser utilizados en otros modelos para abordar detalladamente problemas en el manejo del recurso hídrico, como soporte para la planificación de recursos hídricos balanceando la oferta de agua con la demanda de agua. Con el módulo de escenarios climáticos se puede realizar un análisis de la influencia futura de la precipitación y temperatura. En cuanto a la escorrentía se puede incentivar y aplicar proyectos de Sistemas de Drenaje Sostenible (SuDs) principalmente en la ciudad de Encarnación y contribuyen a la mejora medioambiental de la cuenca y de los ecosistemas receptores. 
Rev. cient. estud. investing. VI Foro de Investigadores; diciembre 2017

Palabras clave: ENOS, balance hídrico, cuenca.

\section{Referencias}

Avila Díaz, Á. J., Carvajal Escobar, Y. \& Gutiérrez Serna, S. E. (2014). Análisis de la influencia de El Niño y La Niña en la oferta hídrica mensual de la cuenca del río Cali. Tecnura, 18(41), 120-133. Recuperado de http://www. scielo.org.co/scielo.php?pid=S0123-921X2014000300010\&script=sci_ abstract\&tlng=es

Dirección Nacional de Aeronaútica Civil y Dirección de Meteorología e Hidrología del Paraguay. (2015). Mapas normales de la precipitaci'on mensual y anual. Recuperado de http://www.meteorologia.gov.py/adm/uploads/ Normales_preci_7100.pdf

Global Consultores. (2015). Balance hídrico cuenca del arroyo Mboi Caé. Encarnación: EBY.

Sokolov, A., Chapman, T. (1981). Métodos de cálculo del balance hídrico. Guía internacional de investigación y métodos. Instituto de Hidrología de España \& UNESCO. Recuperado de http://unesdoc.unesco.org/ images/0013/001377/137771so.pdf

UNECE. (2014). Guía sobre agua y adaptación al cambio climático. Ginebra: Naciones Unidas. 